\title{
Primary Poorly Differentiated Squamous Cell Carcinoma of the Extrahepatic Bile Duct
}

\author{
Takuma Goto $^{1}$, Junpei Sasajima ${ }^{1}$, Kazuya Koizumi ${ }^{1,2}$, Yoshiaki Sugiyama ${ }^{1,3}$, \\ Toru Kawamoto ${ }^{1,4}$, Shugo Fujibayashi ${ }^{1}$, Kentaro Moriichi ${ }^{1}$, Masataka Yamada ${ }^{5}$, \\ Mikihiro Fujiya ${ }^{1}$ and Yutaka Kohgo ${ }^{1}$
}

\begin{abstract}
Squamous cell carcinoma of the extrahepatic bile duct is quite rare. A 77-year-old woman with jaundice and general fatigue was referred to our hospital. Multiphase contrast-enhanced computed tomography visualized a 17-mm solid mass in the junction of the cystic and common bile ducts. The patient underwent pylorus-preserving pancreaticoduodenectomy. The pathological findings demonstrated keratin-positive poorly differentiated squamous cell carcinoma of the extrahepatic bile duct (T3N0M0, stage IIIA). Although adjuvant chemotherapy with gemcitabine was administered, the patient exhibited local recurrence at the site of anastomosis of biliojejunostomy 20 months after resection and died 32 months after resection.
\end{abstract}

Key words: bile duct, carcinoma, squamous cell, chemotherapy, adjuvant, pancreaticoduodenectomy

(Intern Med 55: 1581-1584, 2016)

(DOI: 10.2169/internalmedicine.55.6226)

\section{Introduction}

Extrahepatic bile duct cancer is a malignancy that usually occurs in older patients (1) and is often diagnosed histopathologically as an adenocarcinoma $(2,3)$. In contrast, primary squamous cell carcinoma of the extrahepatic bile duct is quite rare (3); reported cases have included only the differentiated type, and the clinical features remain unknown. We herein report a rare case of primary poorly differentiated squamous cell carcinoma of the extrahepatic bile duct in a 77-year-old Japanese woman who was managed with a pancreaticoduodenectomy and adjuvant chemotherapy.

\section{Case Report}

A 77-year-old woman noticed a yellowish tint to her skin, with itching and general fatigue, and visited a private clinic in July 2009. A biliary plastic stent was inserted to resolve the jaundice, and she was then referred to our hospital for a further evaluation. A physical examination revealed mild icteric skin and sclera without abdominal symptoms. Blood tests showed increased levels of total bilirubin $(2.8 \mathrm{mg} / \mathrm{dL}$; normal range: $0.2-1.0 \mathrm{mg} / \mathrm{dL})$, direct fraction $(2.0 \mathrm{mg} / \mathrm{dL}$; normal range: $0.1-0.6 \mathrm{mg} / \mathrm{dL}$ ), alkaline phosphatase (773 U/ $\mathrm{L}$; normal range: 96-284 U/L), $\gamma$-glutamyl transpeptidase (190 U/L; normal range: 4-32 U/L), aspartate aminotransferase (108 U/L; normal range: 6-40 U/L) and alanine aminotransferase (166 IU/L; normal range: 6-37 U/L), although the white blood cell count was not elevated $(2,660 /$ $\mu \mathrm{L}$; normal range: $3,500-8,500 / \mu \mathrm{L})$ and the C-reactive protein level was only slightly increased $(0.33 \mathrm{mg} / \mathrm{dL}$; normal range: $\leq 0.30 \mathrm{mg} / \mathrm{dL})$. In addition, the levels of cancer antigen 19-9, carcinoembryonic antigen and Dupan-2 were within the normal limits. Multiphase contrast-enhanced computed tomography (CT) scans showed a 17-mm solid mass in the junction of the cystic and common bile ducts (Fig. 1A). The enhancements of the levels of these parame-

${ }^{1}$ Division of Gastroenterology and Hematology/Oncology, Department of Medicine, Asahikawa Medical University, Japan, ${ }^{2}$ Gastroenterology Medicine Center, Shonan Kamakura General Hospital, Japan, ${ }^{3}$ Department of Gastroenterology, Nayoro City General Hospital, Japan, ${ }^{4}$ Department of Gastroenterology, Hokkaido P.W.F.A.C. Asahikawa Kousei General Hospital, Japan and ${ }^{5}$ Department of Gastroenterology, Shibetsu City Hospital, Japan

Received for publication July 21, 2015; Accepted for publication September 6, 2015

Correspondence to Dr. Mikihiro Fujiya, fjym@asahikawa-med.ac.jp 


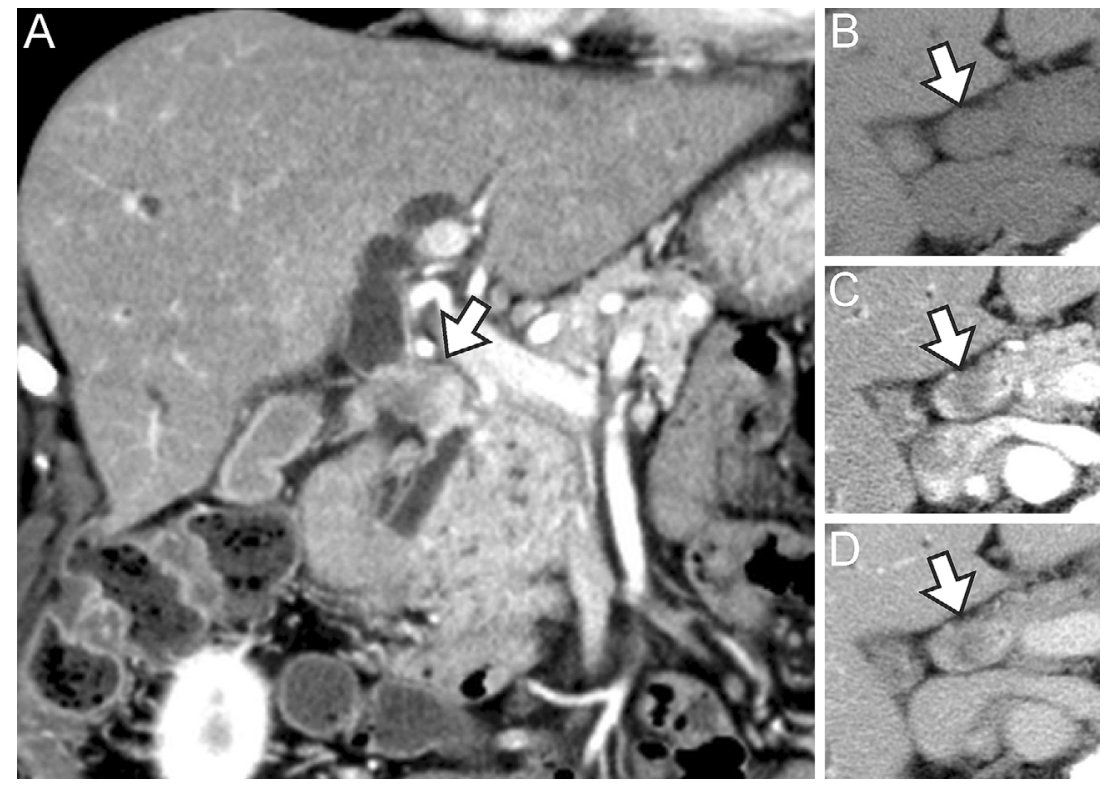

Figure 1. Multiphase contrast-enhanced CT scans. (A) Coronal sections in the early phase demonstrated a 17-mm solid mass (arrow) in the junction of the cystic and common bile ducts. (B-D) Multiphasic CT showed the enhancement of the mass in the early phase to be weak compared with that observed in the liver and pancreas and continued until the delayed phase: (B) plain CT, (C) early phase, (D) delayed phase. The arrow indicates the mass.

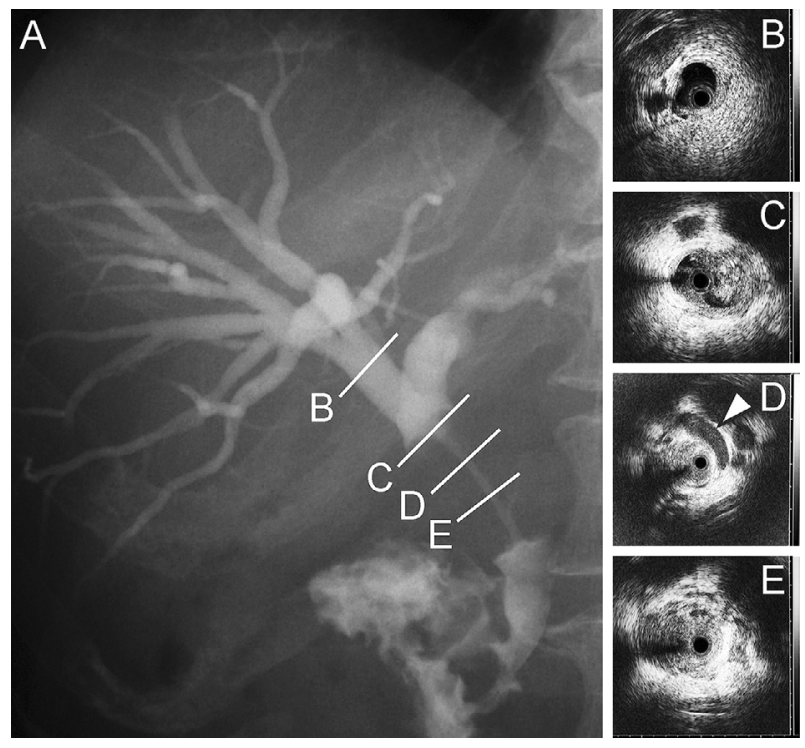

Figure 2. (A) ERCP demonstrated strict and irregular stenosis of the middle portion of the common bile duct. (B-E) IDUS revealed a hypoechoic mass. (B) Both hepatic ducts were intact. (C) The tumor had spread just to the junction of the left and right hepatic ducts. (D) The outer hyperechoic layer of the right hepatic artery (arrowhead) was thin, but not clearly broken, suggesting the possibility of invasion. (E) The tumor was located primarily at the junction of the cystic and common bile ducts.

ters noted in the mass were weak in comparison to those seen in the liver and pancreas in the early and delayed phases (Fig. 1B-D). The small lymph nodes near the com- mon bile duct and proper hepatic artery were enlarged; however, no other metastatic lesions were detected. Endoscopic retrograde cholangiopancreatography (ERCP) demonstrated strict and irregular stenosis of the middle portion of the common bile duct (Fig. 2A), and subsequent intraductal ultrasonography (IDUS) disclosed a hypoechoic mass involving the common bile duct, common hepatic duct and cystic duct (Fig. 2E). The tumor had spread to the junction of the left and right hepatic ducts (Fig. 2C), although both remained intact (Fig. 2B). The outer hyperechoic layer of the bile duct wall on the right hepatic artery (arrowhead) was thin, but not obviously broken, indicating the possibility of partial invasion to the right hepatic artery (Fig. 2D). A specimen obtained from the site of stenosis of the common bile duct via an endoscopy showed p53-positive atypical cells. Although the possibility of tumor invasion into the right hepatic artery was not ruled out, we decided to perform radical removal of the tumor. Because we thought that a hepatopancreaticoduodenectomy was too invasive for this elderly patient with grade 1 performance status, she underwent a pylorus-preserving pancreaticoduodenectomy in August 2009. A pathological examination showed poorly differentiated tumor cells with keratinization and irregular nuclei, whereas no mucin production or ductal formation was detected (Fig. 3A, B). Immunohistochemistry showed the tumor cells to be positive for CK5/6, p53 and MIB-1, but negative for periodic acid staining, indicating poorly differentiated squamous cell carcinoma comprising the entire tumor (Fig. 3C-F). Although visible invasion to the right hepatic artery was not apparent, the tumor microscopically infiltrated the margin to the right hepatic artery (T3NOM0, 

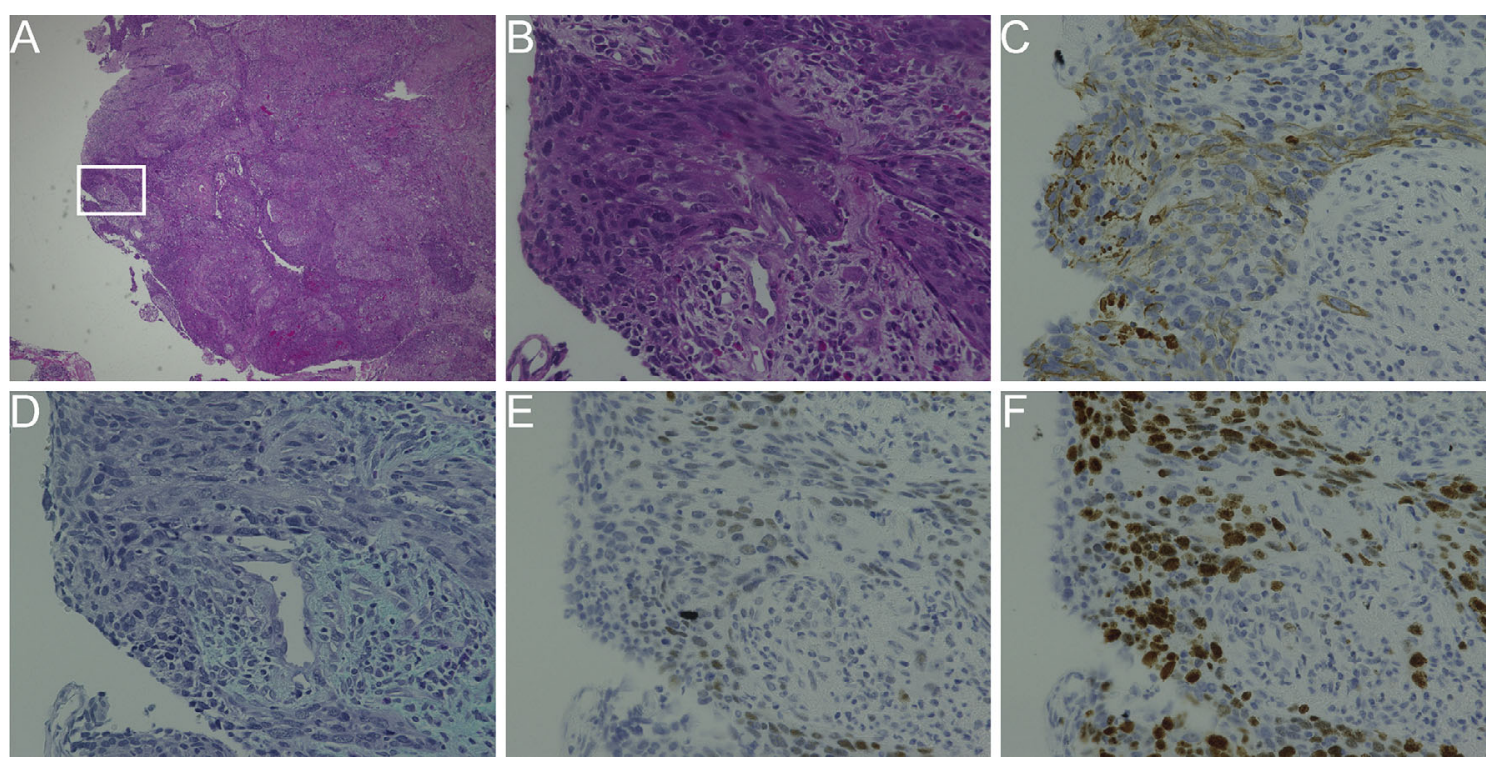

Figure 3. A pathological examination of resected extrabiliary tumor. (A, B) Hematoxylin and Eosin

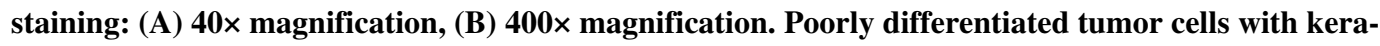
tinization and irregular nuclei, but no mucin production or ductal formation. (C-F) An immunohistochemical analysis (400× magnification). (C) CK5/6, (D) periodic acid staining, (E) p53, (F) MIB-1. Tumor cells were positive for CK5/6, p53 and MIB-1, but negative for periodic acid staining, indicating poorly differentiated squamous cell carcinoma comprising the entire tumor.

stage IIIA). The patient received treatment with gemcitabine (800 mg/body) as adjuvant chemotherapy and exhibited no signs of tumor recurrence for five months after the surgery. Because the adjuvant chemotherapy was often continued for half a year in previous clinical trials for other cancers $(4,5)$, the adjuvant chemotherapy was terminated after the completion of eight courses; however, local recurrence at the site of anastomosis of biliojejunostomy was detected 20 months after the surgery. Gemcitabine was immediately readministered, while the chemotherapy was stopped after four courses due to the progression of dementia with Lewy bodies. The patient died in March 2012 as a result of disease progression caused by local tumor recurrence.

\section{Discussion}

The present report describes a rare case of poorly differentiated squamous carcinoma occurring in the extrahepatic bile duct that was histologically diagnosed based on specimens obtained via an endoscopic biopsy and surgical resection. Extrahepatic bile duct cancer is a relatively rare disease that usually occurs in older patients, with a poor prognosis and an estimated incidence of less than 2 per 100,000 (1). More than $90 \%$ of all cases of extrahepatic cancer involve adenocarcinomas (2), as with the other malignancies of the biliary tract. In contrast, squamous cell carcinoma of the extrahepatic bile duct is quite rare (3), with only nine cases reported in the English literature over approximately 100 years (Table) (6-14). All patients, including our patient, complained of jaundice. Abdominal pain, fever and body weight loss were also observed in some cases, indicating that the symptoms of squamous cell carcinoma are not significantly different from those of adenocarcinoma in the extrahepatic bile duct. In the present case, the tumor was poorly enhanced on both early- and delayed-phase multiphase CT, whereas approximately $80 \%$ of extrahepatic bile duct cancers show enhancement in the arterial and/or portal phases on CT (15). Among these case reports, three authors mentioned CT findings (11-13), among which only one case was associated with CT images showing an enhanced mass of well-differentiated squamous cell carcinoma (14). The feature of contrast enhancement seen in our case may reflect the severe sclerotic nature of this lesion with poor differentiation.

IDUS is a useful option for local staging to select patients who may benefit from surgical resection (16). The current case is the first to involve features of IDUS of extrahepatic squamous cell carcinoma. In this case, IDUS showed the outer hyperechoic layer of the right hepatic artery to be thin, but not obviously broken. Indeed, visible invasion to the right hepatic artery was not detected, although microscopic infiltration of the margin to the right hepatic artery was histopathologically suspected in the surgical specimen. IDUS was therefore useful for determining the local extent of tumor invasion in this case.

Based on the findings of multiphase CT and IDUS, the patient received pancreaticoduodenectomy and adjuvant chemotherapy. Among the nine case reports, for six cases, the histological grade was mentioned, namely, "well" in three cases $(8,11,14)$, "moderate" in two cases $(7,12)$ and "moderate to poor" in one case (13). The present paper may be the first report of completely poorly differentiated 
Table. Case Reports of Primary Squamous Cell Carcinoma in the Extrahepatic Bile Duct.

\begin{tabular}{|c|c|c|c|c|c|c|c|c|c|}
\hline References & Year & Age & Sex & Location & $\begin{array}{l}\text { UICC } \\
\text { Stage }\end{array}$ & $\begin{array}{l}\text { Differen } \\
\text {-tiation }\end{array}$ & $\begin{array}{l}\text { CT } \\
\text { findings }\end{array}$ & Treatment & $\begin{array}{l}\text { Survival } \\
\text { (Month) }\end{array}$ \\
\hline 6 & 1930 & 58 & $\mathrm{M}$ & Perihilar & $\mathrm{IVb}$ & N/A & $\mathrm{N} / \mathrm{A}$ & None & 1 \\
\hline 7 & 1978 & 29 & $\mathrm{~F}$ & Cyst Junc & IB & Mod & N/A & $\mathrm{PD}+\mathrm{Ch}$ & 8 \\
\hline 8 & 1979 & 68 & $\mathrm{M}$ & $\begin{array}{l}\text { mid } \\
\text { portion }\end{array}$ & NA & Well & N/A & CCE & 6 \\
\hline 9 & 1980 & 56 & $\mathrm{~F}$ & Perihilar & IVB & N/A & N/A & $\begin{array}{l}\mathrm{CCE}+\mathrm{RT}+ \\
\text { T-tube }\end{array}$ & 3 \\
\hline 10 & 2004 & 86 & $\mathrm{~F}$ & Cyst Junc & N/A & N/A & N/A & $\begin{array}{l}\text { CCE+RT+EM } \\
\text { S }\end{array}$ & 18 \\
\hline 11 & 2005 & 60 & M & distal & IB & Well & $\begin{array}{l}\text { thickening of the } \\
\text { distal bile duct wall }\end{array}$ & PD & N/A \\
\hline 12 & 2008 & 28 & $\mathrm{~F}$ & Perihilar & N/A & Mod & $\begin{array}{l}\text { dilation of the left } \\
\text { bile duct }\end{array}$ & $\begin{array}{l}\text { L hep } \\
\text { lobe+RT }\end{array}$ & 18(Alive) \\
\hline 13 & 2008 & 41 & $\mathrm{~F}$ & Perihilar & IVA & Mod-por & N/A & Bypass+EMS & N/A \\
\hline 14 & 2011 & 66 & $\mathrm{M}$ & Perihilar & IVA & well & $\begin{array}{l}\text { enhanced mass in } \\
\text { hilar bile duct }\end{array}$ & $\begin{array}{l}\text { R hep lobe } \\
+\mathrm{Ch}\end{array}$ & 12 \\
\hline Our case & 2014 & 77 & $\mathrm{~F}$ & Cyst Junc & IIIA & por & $\begin{array}{l}\text { poorly enhanced } \\
\text { solid mass }\end{array}$ & $\mathrm{PD}+\mathrm{Ch}$ & 32 \\
\hline
\end{tabular}

Cyst junc: junction of the cystic and common bile ducts, Mod: moderate differentiation, Por: poor differentiation, PD: pancreaticoduodenectomy, Ch: chemotherapy, CCE: cholecystectomy, RT: radiation therapy, EMS: endoscopic metallic stent, L hep lobe: extended left hepatic lobectomy, $\mathrm{R}$ hep lobe: extended right hepatic lobectomy, N/A: not available

squamous cell carcinoma of the extrahepatic bile duct.

A radical resection was performed in four cases $(7,11,12,14)$; however, the use of adjuvant chemotherapy has not been previously demonstrated. Therefore, the present case is also the first to be reported in which adjuvant chemotherapy was performed for squamous cell carcinoma of the extrahepatic bile duct. Although there is no universally agreed upon standard chemotherapy regimen for patients with extrahepatic bile duct cancer (17), a relatively low dose of gemcitabine was selected in this case because this drug is frequently used to treat bile duct cancer. Consequently, our patient survived for 32 months, which is much longer than that observed in previously reported cases (6-14), and her symptoms were well managed with a good quality of life.

We herein described a rare case of primary squamous cell carcinoma of the extrahepatic bile duct. This may be the first case of poorly differentiated squamous cell carcinoma in which adjuvant chemotherapy was performed, which subsequently prolonged the patient's survival.

The authors state that they have no Conflict of Interest (COI).

\section{References}

1. Khan SA, Thomas HC, Davidson BR, Taylor-Robinson SD. Cholangiocarcinoma. Lancet 366: 1303-1314, 2005.

2. Cleary SP, Dawson LA, Knox JJ, Gallinger S. Cancer of the gallbladder and extrahepatic bile ducts. Curr Probl Surg 44: 396-482, 2007.

3. Sako K, Seitzinger GL, Garside E. Carcinoma of the extrahepatic bile ducts; review of the literature and report of six cases. Surgery 41: 416-437, 1957.

4. Oettle H, Neuhaus P, Hochhaus A, et al. Adjuvant chemotherapy with gemcitabine and long-term outcomes among patients with resected pancreatic cancer: the CONKO-001 randomized trial. JAMA 310: 1473-1481, 2013.

5. Maeda A, Boku N, Fukutomi A, et al. Randomized phase III trial of adjuvant chemotherapy with gemcitabine versus S-1 in patients with resected pancreatic cancer: Japan Adjuvant Study Group of Pancreatic Cancer (JASPAC-01). Jpn J Clin Oncol 38: 227-229, 2008.

6. Cabot R. Case 16261: four months' jaundice and rectal pain. N Engl J Med 202: 1260-1262, 1930.

7. Burger RE, Meeker WR, Luckett PM. Squamous cell carcinoma of the common bile duct. Southern medical journal 71: 2: 216219, 1978.

8. Gulsrud PO, Feinberg M, Koretz RL. Rapid development of cirrhosis secondary to squamous cell carcinoma of the common bile duct. Dig Dis Sci 24: 166-169, 1979.

9. Aranha GV, Reyes CV, Greenlee HB, Field T, Brosnan J. Squamous cell carcinoma of the proximal bile duct: a case report. J Surgl Oncol 15: 1: 29-35, 1980.

10. Gatof D, Chen YK, Shah RJ. Primary squamous cell carcinoma of the bile duct diagnosed by transpapillary cholangioscopy: case report and review. Gastrointest Endosc 60: 2: 300-304, 2004.

11. Sewkani A, Kapoor S, Sharma S, et al. Squamous cell carcinoma of the distal common bile duct. JOP 6: 162-165, 2005.

12. Abbas R, Willis J, Kinsella T, Siegel C, Sanabria J. Primary squamous cell carcinoma of the main hepatic bile duct. Can $\mathbf{J}$ Surg 51: E85-E86, 2008.

13. Price L, Kozarek R, Agoff N. Squamous cell carcinoma arising within a choledochal cyst. Dig Dis Sci 53: 2822-2825, 2008.

14. Yamana I, Kawamoto S, Nagao S, Yoshida $T$, Yamashita $Y$. Squamous cell carcinoma of the hilar bile duct. Case Rep Gastroenterol 5: 463-470, 2011.

15. Ayuso JR, Pages M, Darnell A. Imaging bile duct tumors: staging. Abdom Imaging 38: 1071-1081, 2013.

16. Khashab MA, Fockens P, Al-Haddad MA. Utility of EUS in patients with indeterminate biliary strictures and suspected extrahepatic cholangiocarcinoma (with videos). Gastrointest Endosc 76: 1024-1033, 2012.

17. Sun TT, Wang JL, Fang JY. Gemcitabine alone or in combination with Cisplatin for advanced biliary tract carcinomas: an overview of clinical evidence. Asian Pac J Cancer Prev 14: 877-883, 2013.

(C) 2016 The Japanese Society of Internal Medicine http://www.naika.or.jp/imonline/index.html 